\title{
On the Noncommutative and Nonassociative Geometry of Octonionic Spacetime, Modified Dispersion Relations and Grand Unification
}

\author{
Carlos Castro \\ Center for Theoretical Studies of Physical Systems \\ Clark Atlanta University, Atlanta, GA. 30314, castro@ctsps.cau.edu
}

April 2007, Revised May 2007

\begin{abstract}
The Octonionic Geometry (Gravity) developed long ago by Oliveira and Marques is extended to Noncommutative and Nonassociative Spacetime coordinates associated with octonionic-valued coordinates and momenta. The octonionic metric $\mathbf{G}_{\mu \nu}$ already encompasses the ordinary spacetime metric $g_{\mu \nu}$, in addition to the Maxwell $U(1)$ and $S U(2)$ Yang-Mills fields such that implements the Kaluza-Klein Grand Unification program without introducing extra spacetime dimensions. The color group $S U(3)$ is a subgroup of the exceptional $G_{2}$ group which is the automorphism group of the octonion algebra. It is shown that the flux of the $S U(2)$ Yang-Mills field strength $\overrightarrow{\mathcal{F}}_{\mu \nu}$ through the areamomentum $\vec{\Sigma}^{\mu \nu}$ in the internal isospin space yields corrections $O\left(1 / M_{\text {Planck }}^{2}\right)$ to the energy-momentum dispersion relations without violating Lorentz invariance as it occurs with Hopf algebraic deformations of the Poincare algebra. The known Octonionic realizations of the Clifford $C l(8), C l(4)$ algebras should permit the construction of octonionic string actions that should have a correspondence with ordinary string actions for strings moving in a curved Clifford-space target background associated with a $C l(3,1)$ algebra.
\end{abstract}

Keywords: Nonassociative Geometry, Clifford algebras, Quaternions, Octonionic Gravity, Unification, Strings. 


\section{Introduction}

Exceptional, Jordan, Division, Clifford and Noncommutative algebras are deeply related and essential tools in many aspects in Physics, see for instance [1], [2], [3], [4], [5], [6], [7]., [9], [8], [36], [11], [15], [13], [14], [16], [24]. [29], [56], [35]. Ever since the discovery $[58]$ that $11 D$ supergravity, when dimensionally reduced to an $n$-dim torus led to maximal supergravity theories with hidden exceptional symmetries $E_{n}$ for $n \leq 8$, it has prompted intensive research to explain the higher dimensional origins of these hidden exceptional $E_{n}$ symmetries. More recently, there has been a lot of interest in the infinite-dim hyperbolic Kac-Moody $E_{10}$ and non-linearly realized $E_{11}$ algebras arising in the asymptotic chaotic oscillatory solutions of Supergravity fields close to cosmological singularities [57]

The classification of symmetric spaces associated with the scalars of $N$ extended Supergravity theories, emerging from compactifications of $11 D$ supergravity to lower dimensions, and the construction of the $U$-duality groups as spectrum-generating symmetries for four-dimensional BPS black-holes [27], [34] also involved exceptional symmetries associated with the exceptional magic Jordan algebras $J_{3}[R, C, H, O]$. The discovery of the anomaly free 10-dim heterotic string for the algebra $E_{8} \times E_{8}$ was another hallmark of the importance of Exceptional Lie groups in Physics.

Exceptional Jordan Matrix Models based on the compact $E_{6}$ involve a double number of the required physical degrees of freedom inherent in a complex-valued action [11]. This led Ohwashi to construct an interacting pair of mirror universes within the compact $E_{6}$ matrix model and equipped with a $S p(4, \mathbf{H}) / Z_{2}$ symmetry based on the quaternionic valued symplectic group. The interacting picture resembles that of the bi-Chern-Simons gravity models. The complex counterpart of the Chern-Simon-Witten theory has been studied by [62] where the complex (holomorphic) analogue of the Gauss linking number for complex curves embedded in a Calabi-Yau threefold was defined. A nonassociative formulation of bosonic strings in $D=26$ using Jordan algebras was presented a while back by [35]. A construction of nonassociative Chern-Simons membranes and 3-branes based on the large $N$ limit of Exceptional Jordan algebras was put forward by $[12]$.

Some recent developments related to Jordan exceptional algebras and octonions have been found. The $E_{7}$ Cartan quartic invariant was used by [32] to construct the entanglement measure associated with the tripartite entanglement of seven quantum-bits represented by the group $S L(2, C)^{3}$ and realized in terms of $2 \times 2 \times 2$ cubic matrices. It was shown by [33] that this tripartite entanglement of seven quantum-bits is entirely decoded into the discrete geometry of the octonion Cayley-Fano plane. The analogy between quantum information theory and supersymmetric black holes in $4 d$ string theory compactifications was extended further by [33]. The role of Jordan algebras associated with the homogeneous symmetric spaces present in the study of extended supergravities, BPS black holes, quantum attractor flows and automorphic forms can be found 
in [27].

The $E_{8}$ group was proposed long ago [26] as a candidate for a grand unification model building in $D=4$. The supersymmetric $E_{8}$ model has more recently been studied as a fermion family and grand unification model [26] under the assumption that there is a vacuum gluino condensate but this condensate is not accompanied by a dynamical generation of a mass gap in the pure $E_{8}$ gauge sector. A study of the interplay among Exceptional Groups, del Pezzo surfaces and the extra massless particles arising from rational double point singularities can be found in [44], [43] . Clifford algebras and $E_{8}$ are key ingredients in Smith's $D_{4}-D_{5}-E_{6}-E_{7}-E_{8}$ grand unified model in $D=8$ [17].

A novel Chern-Simons $E_{8}$ gauge theory of Gravity in $D=15$ based on an octic $E_{8}$ invariant expression in $D=16$ (recently constructed by Cederwall and Palmkvist) was developed by [47]. A grand unification model of gravity with the other forces is very plausible within the framework of a supersymmetric extension (to incorporate spacetime fermions) of this Chern-Simons $E_{8}$ gauge theory. An $E_{8}$ gauge bundle formulation was instrumental in understanding the topological part of the 11-dim $M$-theory partition function. The nature of this 11-dim $E_{8}$ gauge theory remains unknown. The Chern-Simons $E_{8}$ gauge theory of Gravity in $D=15$ may shed some light into solving this problem after a dimensional reduction.

A complexification of ordinary gravity (not to be confused with HermitianKahler geometry ) has been known for a long time. Complex gravity requires that $g_{\mu \nu}=g_{(\mu \nu)}+i g_{[\mu \nu]}$ so that now one has $g_{\nu \mu}=\left(g_{\mu \nu}\right)^{*}$, which implies that the diagonal components of the metric $g_{z_{1} z_{1}}=g_{z_{2} z_{2}}=g_{\tilde{z}_{1} \tilde{z}_{1}}=g_{\tilde{z}_{2} \tilde{z}_{2}}$ must be real. A treatment of a non-Riemannan geometry based on a complex tangent space and involving a symmetric $g_{(\mu \nu)}$ plus antisymmetric $g_{[\mu \nu]}$ metric component was first proposed by Einstein-Strauss [10] (and later on by [19] ) in their unified theory of Electromagentism with gravity by identifying the EM field strength $F_{\mu \nu}$ with the antisymmetric metric $g_{[\mu \nu]}$ component.

Borchsenius [18] formulated the quaternionic extension of Einstein-Strauss unified theory of gravitation with EM by incorporating appropriately the $S U(2)$ Yang-Mills field strength into the degrees of a freedom of a quaternionc-valued metric. Oliveira and Marques [20] later on provided the Octonionic Gravitational extension of Borchsenius theory involving two interacting $S U(2)$ YangMills fields and where the exceptional group $G_{2}$ was realized naturally as the automorphism group of the octonions.

Ashtekar's formulation of Gravity in $D=12$ dimensions using octonionic structure constants has been formulated by [40]. Modified dispersion relations based on the simplest Octonionic geometry has been discussed in a very different context than the work presented here by [41]. The (para) octonionic geometry of the projective plane has been analyzed extenisvely by [42]. Octonionc spinors have been studied by [28], [46], among others. The bar operations and hyper-complex group theory to formulate a consistent Octonionic QM have been developed by [45]. The non-Desarguesian geometry of the Moufang projective plane to describe Octonionic QM was discussed by [15].

It was shown in [21] how one could generalize Octonionic Gravitation into 
an Extended Relativity theory in Clifford spaces, involving poly-vector valued (Clifford-algebra valued) coordinates and fields, where in addition to the speed of light there is also an invariant length scale (set equal to the Planck scale) in the definition of a generalized metric distance in Clifford spaces encoding, lengths, areas, volumes and hyper-volumes metrics. An overview of the basic features of the Extended Relativity in Clifford spaces can be found in [21]. $E_{8}$ Yang-Mills theory can naturally be embedded into a $C l(16)$ algebra Gauge Theory [48] and the $11 D$ Chern-Simons (Super) Gravity [60] is a very small sector of a more fundamental polyvector-valued gauge theory in Clifford spaces. Polyvector-valued Supersymmetries [61] in Clifford-spaces [51] turned out to be more fundamental than the supersymmetries associated with $M, F$ theory superalgebras.

Generalized Complex geometry was developed by Hitchin and involves a metric and a two-form, an antisymmetric field $B_{\mu \nu}$ ( not the same as $g_{[\mu \nu]}$ ) and plays an important role in string theory compactifications with flux. Recently Hitchin's geometry has been generalized to manifolds with a metric and $p$ forms by [30] as the appropriate geometry for $M$ theory. Generalized Complex geometry has also been instrumental in the Geometric Langlands Program in Physics advanced by [31].

The purpose of this work is is to advance further the Octonionic Geometry (Gravity) of [20] by enlarging the ordinary spacetime coordinates to octonionicvalued coordinates furnishing a natural realization of a Noncommutative and Nonassociative spacetime. The most salient feature of the decomposition of the split-octonionic metric $\mathbf{G}_{\mu \nu}$ is that it already encompasses the ordinary spacetime metric $g_{\mu \nu}$, in addition to the Maxwell EM field and $S U(2)$ YangMills fields. Therefore, it automatically implements the Kaluza-Klein program without introducing extra spacetime dimensions. Clifford algebras have been used by [17], [22], [23], among others to attain unification without extra spacetime dimensions. It is shown that the flux of $\overrightarrow{\mathcal{F}}_{\mu \nu}$ through the area-momentum $\vec{\Sigma}^{\mu \nu}$ in the internal isospin space yields the corrections $O\left(1 / M_{\text {Planck }}^{2}\right)$ to the energy-momentum dispersion relations.

The automorphism group of the octonionic algebra is the 14-dim exceptional $G_{2}$ group that admits a $S U(3)$ subgroup leaving invariant the idempotents $u_{o}, u_{o}^{*}$ of the split-octonionic algebra. This $S U(3)_{c}$ was identified as the color group acting on the quarks and antiquarks triplets [15] $\Psi_{\alpha}=u_{i} \Psi_{\alpha}^{i}, \bar{\Psi}_{\alpha}=-u_{i}^{*} \bar{\Psi}_{\alpha}^{i}, i=$ $1,2,3$, respectively. Octonionic realizations of the Clifford $\mathrm{Cl}(8), \mathrm{Cl}(4)$ algebras should permit the construction of octonionic strings where the $1+1$-octonionicdim worldsheet of an octonionic string has a correspondence with an $8+8$ real-dimensional spacetime of split signature corresponding to the $C l(4)$-space. For all these reasons we deem very important to explore further the novelties behind the octonionic geometry developed here within the framework of the unification of gravity with the other forces and the role played by the Planck scale in the modifications of the dispersion relations without violating Lorentz invariance. 


\section{Octonionic Geometry of Noncommutative and Nonassociative Spacetime}

Given an octonion $\mathbf{X}$ it can be expanded in a basis $\left(e_{o}, e_{m}\right)$ as

$$
\mathbf{X}=x^{o} e_{o}+x^{m} e_{m}, \quad m, n, p=1,2,3, \ldots . .7 .
$$

where $e_{o}$ is the identity element. The Noncommutative and Nonassociative algebra of octonions is determined from the relations

$$
e_{o}^{2}=e_{o}, e_{o} e_{i}=e_{i} e_{o}=e_{i}, e_{i} e_{j}=-\delta_{i j} e_{o}+c_{i j k} e_{k}, i, j, k=1,2,3, \ldots .7 .
$$

where the fully antisymmetric structure constants $c_{i j k}$ are taken to be 1 for the combinations $(123),(516),(624),(435),(471),(673),(672)$. The octonion conjugate is defined

$$
\overline{\mathbf{X}}=x^{o} e_{o}-x^{m} e_{m}
$$

and the norm

$$
N(\mathbf{X})=\langle\mathbf{X} \mathbf{X}\rangle=\operatorname{Real}(\overline{\mathbf{X}} \mathbf{X})=\left(x_{o} x_{o}+x_{k} x_{k}\right) .
$$

The inverse

$$
\mathbf{X}^{-1}=\frac{\overline{\mathbf{X}}}{N(\mathbf{X})}, \quad \mathbf{X}^{-1} \mathbf{X}=\mathbf{X X}^{-1}=1 .
$$

The non-vanishing associator is defined by

$$
\{\mathbf{X}, \mathbf{Y}, \mathbf{Z}\}=(\mathbf{X Y}) \mathbf{Z}-\mathbf{X}(\mathbf{Y Z})
$$

In particular, the associator

$$
\left\{e_{i}, e_{j}, e_{k}\right\}=d_{i j k l} e_{l}, \quad d_{i j k l}=\epsilon_{i j k l m n p} c^{m n p}, i, j, k \ldots=1,2,3, \ldots . .7
$$

The generators of the split-octonionic algebra admit a realization in terms of the $4 \times 4$ Zorn matrices (in blocks of $2 \times 2$ matrices) by writing

$$
\begin{array}{ccc}
u_{o}=\frac{1}{2}\left(e_{o}+i e_{7}\right), & u_{o}^{*}=\frac{1}{2}\left(e_{o}-i e_{7}\right) \\
u_{i}=\frac{1}{2}\left(e_{i}+i e_{i+3}\right), & u_{i}^{*}=\frac{1}{2}\left(e_{i}-i e_{i+3}\right) \\
u_{o}=\left(\begin{array}{cc}
0 & 0 \\
0 & \omega_{0}
\end{array}\right) & u_{o}^{*}=\left(\begin{array}{cc}
\omega_{o} & 0 \\
0 & 0
\end{array}\right)
\end{array}
$$




$$
u_{i}=\left(\begin{array}{cc}
0 & 0 \\
\omega_{i} & 0
\end{array}\right) \quad u_{i}^{*}=\left(\begin{array}{cc}
0 & -\omega_{i} \\
0 & 0
\end{array}\right)
$$

The quaternionic generators $\omega_{o}, \omega_{i}, i=1,2,3$ obey the algebra $\omega_{i} \omega_{j}=\epsilon_{i j k} \omega_{k}-$ $\delta_{i j} \omega_{o}$ and are related to the Pauli spin $2 \times 2$ matrices by setting $\sigma_{i}=i \omega_{i}$ and $\omega_{o}=\mathbf{1}_{2 \times 2}$. The $u_{i}, u_{i}^{*}$ behave like fermionic creation and annihilation operators corresponding to an exceptional (non-associative) Grassmannian algebra

$$
\begin{gathered}
\left\{u_{i}, u_{j}\right\}=\left\{u_{i}^{*}, u_{j}^{*}\right\}=0, \quad\left\{u_{i}, u_{j}^{*}\right\}=-\delta_{i j} . \\
\frac{1}{2}\left[u_{i}, u_{j}\right]=\epsilon_{i j k} u_{k}^{*}, \quad \frac{1}{2}\left[u_{i}^{*}, u_{j}^{*}\right]=\epsilon_{i j k} \quad u_{k}, \quad u_{o}^{2}=u_{o}, \quad\left(u_{o}^{*}\right)^{2}=u_{o}^{*} .
\end{gathered}
$$

Unlike the octonionic algebra, the split-octonionic algebra contains zero divisors and therefore is not a division algebra.

The automorphism group of the octonionic algebra is the 14-dim exceptional $G_{2}$ group that admits a $S U(3)$ subgroup leaving invariant the idempotents $u_{o}, u_{o}^{*}$. This $S U(3)_{c}$ was identified as the color group acting on the quarks and antiquarks triplets [15] $\Psi_{\alpha}=u_{i} \Psi_{\alpha}^{i}, \bar{\Psi}_{\alpha}=-u_{i}^{*} \bar{\Psi}_{\alpha}^{i}, i=1,2,3$, respectively. From the split-octonionic algebra multiplication table one learns that triplet $\times$ triplet $=$ anti triplet and triplet $\times$ anti triplet $=$ singlet providing a very natural algebraic interpretation of confinement of 3 quarks. An effective dynamical SUSY and the phenomenological manifestation of quark dynamics inside hadrons leading to the classification of mesons an baryons can be found in [16], [5] and references therein.

The Zorn matrix product of

$$
\mathbf{A}=\left(\begin{array}{cc}
A_{o} \omega_{o} & -A_{i} \omega^{i} \\
B_{i} \omega^{i} & B_{o} \omega_{o}
\end{array}\right) \quad \mathbf{B}=\left(\begin{array}{cc}
C_{o} \omega_{o} & -C_{i} \omega^{i} \\
D_{i} \omega^{i} & D_{o} \omega_{o}
\end{array}\right)
$$

is defined by

$$
\mathbf{A} \bullet \mathbf{B}=\left(\begin{array}{cc}
\left(A_{o} C_{o}+A_{i} D_{i}\right) \omega_{o} & -\left(A_{o} C_{k}+D_{o} A_{k}+\epsilon_{i j k} B_{i} D_{j}\right) \omega^{k} \\
\left(C_{o} B_{k}+B_{o} D_{k}+\epsilon_{i j k} A_{i} C_{j}\right) \omega^{k} & \left(B_{o} D_{o}+B_{i} C_{i}\right) \omega_{o}
\end{array}\right)
$$

where we have used

$$
\begin{gathered}
\omega_{i} \omega_{j}=\epsilon_{i j k} \omega_{k}-\delta_{i j} \omega_{o} \Rightarrow \omega_{i} \omega_{i}=-\omega_{o}, \text { for each } i=1,2,3 \Rightarrow \\
\vec{x} . \vec{y}=\left(x_{i} \omega_{i}\right)\left(y_{i} \omega_{i}\right)=-x_{i} y_{i} \omega_{o} .
\end{gathered}
$$

the last minus sign must be kept in my mind in the products that follow. The multiplication product of the split-octonions generators $u_{o}, u_{o}^{*}, u_{i}, u_{i}^{*}$ is reproduced in this Zorn matrix realization. 
The split-octonionic-valued spacetime vector $\mathbf{Z}^{\mu}, \mu=1,2,3, \ldots \ldots, D$ can be represented in terms of the $4 \times 4$ (spacetime vector-valued) Zorn matrix $Z_{a b}^{\mu}$ as

$$
\mathbf{Z}^{\mu}=x_{o}^{\mu} u_{o}^{*}+y_{o}^{\mu} u_{o}+x_{i}^{\mu} u_{i}^{*}+y_{i}^{\mu} u_{i}=\left(\begin{array}{ccc}
x_{o}^{\mu} \omega_{o} & -x_{i}^{\mu} \omega^{i} \\
y_{i}^{\mu} \omega^{i} & y_{o}^{\mu} \omega_{o}
\end{array}\right)
$$

$x_{o}^{\mu}, x_{k}^{\mu}, y_{o}^{\mu}, y_{k}^{\mu}$ are real valued variables. When the spacetime metric is $\eta_{\mu \nu} \mathbf{1}_{4 \times 4}$, the bilinear form

$$
\begin{gathered}
\frac{1}{4} \operatorname{Trace}\left[\eta_{\mu \nu} \mathbf{Z}^{\mu} \bullet \mathbf{Z}^{\nu}\right]=\frac{1}{4} \eta_{\mu \nu}\left[x_{o}^{\mu} x_{o}^{\nu}+y_{o}^{\mu} y_{o}^{\nu}+x_{k}^{\mu} y_{k}^{\nu}+x_{k}^{\nu} y_{k}^{\mu}\right] \text { Trace }\left[\mathbf{1}_{2 \times 2}\right]= \\
\frac{1}{2} \eta_{\mu \nu}\left[x_{o}^{\mu} x_{o}^{\nu}+y_{o}^{\mu} y_{o}^{\nu}+x_{k}^{\mu} y_{k}^{\nu}+x_{k}^{\nu} y_{k}^{\mu}\right]
\end{gathered}
$$

furnishes the analog of an inner product. We may notice that when $x_{o}^{\mu}=y_{o}^{\mu}$, the fist two terms of the last expression combine to agree with the standard quadratic norm of a vector in Minkowski spacetime $\eta_{\mu \nu} x_{o}^{\mu} x_{o}^{\nu}$.

The octononic conjugation is defined by $e_{o} \rightarrow e_{o}$ and $e_{m} \rightarrow-e_{m}$, so

$$
\overline{\mathbf{Z}}^{\mu}=x_{o}^{\mu} u_{o}+y_{o}^{\mu} u_{o}^{*}-x_{i}^{\mu} u_{i}^{*}-y_{i}^{\mu} u_{i}=\left(\begin{array}{ccc}
y_{o}^{\mu} \omega_{o} & x_{i}^{\mu} \omega^{i} \\
-y_{i}^{\mu} \omega^{i} & x_{o}^{\mu} \omega_{o}
\end{array}\right)
$$

When $x_{o}^{\mu}, y_{o}^{\mu}, x_{i}^{\mu}, y_{i}^{\mu}$ are complex valued one may define the "Hermitian" conjugate by taking the complex conjugate of the components of $\overline{\mathbf{Z}}^{\mu}$ :

$$
\left(\mathbf{Z}^{\mu}\right)^{\dagger}=\left(x_{o}^{\mu}\right)^{*} u_{o}+\left(y_{o}^{\mu}\right)^{*} u_{o}^{*}-\left(x_{i}^{\mu}\right)^{*} u_{i}^{*}-\left(y_{i}^{\mu}\right)^{*} u_{i}=\left(\begin{array}{cc}
\left(y_{o}^{\mu}\right)^{*} \omega_{o} & \left(x_{i}^{\mu}\right)^{*} \omega^{i} \\
-\left(y_{i}^{\mu}\right)^{*} \omega^{i} & \left(x_{o}^{\mu}\right)^{*} \omega_{o}
\end{array}\right)
$$

It should be emphasized that the dagger operation $\dagger$ must not be confused with the Hermitian adjoint operation but it is defined by taking the complex conjugate of the components of $\overline{\mathbf{Z}}^{\mu}$.

The split-octonionic-valued metric $\mathbf{G}_{\mu \nu}=G_{\mu \nu}^{a b}$ obeying $\left(\mathbf{G}_{\mu \nu}\right)^{\dagger}=\mathbf{G}_{\nu \mu}$ can be represented by a $4 \times 4$ (tensor-valued) Zorn matrix as [20]

$$
\begin{gathered}
\mathbf{G}_{\mu \nu}=\left(\begin{array}{cc}
\left(g_{\mu \nu}+i g_{[\mu \nu]}\right) \omega_{o} & -s_{[\mu \nu]}^{i} \omega_{i} \\
r_{[\mu \nu]}^{i} \omega_{i} & \left(g_{\mu \nu}+i g_{[\mu \nu]}\right) \omega_{o}
\end{array}\right)= \\
\left(\begin{array}{cc}
\left(g_{\mu \nu}+i \kappa^{2} F_{\mu \nu}\right) \omega_{o} & \kappa^{2} \lambda^{2} \mathcal{F}_{\mu \nu i}^{(1)} \omega^{i} \\
-\kappa^{2} \lambda^{2} \mathcal{F}_{\mu \nu i}^{(2)} \omega^{i} & \left(g_{\mu \nu}+i \kappa^{2} F_{\mu \nu}\right) \omega_{o}
\end{array}\right), \quad\left(\mathbf{G}_{\mu \nu}\right)^{\dagger}=\mathbf{G}_{\nu \mu}
\end{gathered}
$$


$F_{\mu \nu}$ is the Maxwell $U(1)$ valued electromagnetic field strength. $\mathcal{F}_{\mu \nu}^{(1)}=\mathcal{F}_{\mu \nu i}^{(1)} \omega^{i}$ and $\mathcal{F}_{\mu \nu}^{(2)}=\mathcal{F}_{\mu \nu i}^{(2)} \omega^{i}$ are two $S U(2)$ valued field strengths of the Yang-Mills type corresponding to an internal $S U(2)_{L} \times S U(2)_{R}$ symmetry.

The length parameter $\kappa$ is defined

$$
\kappa^{2}=4 \pi \frac{L_{P}^{2}}{e^{2}}, \Rightarrow \kappa=2 \sqrt{137 \pi} L_{\text {Planck }} \sim 4.15 \times 10^{-32} \mathrm{~cm} .
$$

in $\hbar=c=1$ units and $\lambda=-e / \mathcal{Q}$ is the ratio of the electric charge $e$ and the isotopic charge $\mathcal{Q}$. The most salient feature of the decomposition of the split-octonionic metric $\mathbf{G}_{\mu \nu}$ in the form (17) is that it already encompasses the ordinary spacetime metric $g_{\mu \nu}$, in addition to the Maxwell EM field and $S U(2)$ Yang-Mills fields. Therefore, it automatically implements the KaluzaKlein program without introducing extra spacetime dimensions! A realization of this idea appears also in Smith's Unified model of gravity with all the other forces based on the Cliff $(8)$ algebra [17] and in the Cliff $(4)$ algebra spin gauge theory model of [22], for example. The geometric basis of the Standard Model based on Clifford algebras can be found in [23].

When the spacetime is four-dimensional, when one takes into account the internal directions $0,1,2,3$ corresponding to the generators $\omega_{o}, \omega_{1}, \omega_{2}, \omega_{3}$ one has an effective $4+4=8$-dim space. A unified model involving an 8-dim space based on the Clifford algebra $\mathrm{Cl}(8)$ and octonions has been constructed by Smith [17]. A generalization of Smith's model to C-spaces was advanced in [49].

Despite the fact that the octonions are non-associative the Zorn matrix product does satisfy the cyclic trace property

$$
\operatorname{Trace}[\mathbf{A} \bullet \mathbf{B}]=\operatorname{Trace}[\mathbf{B} \bullet \mathbf{A}] \text {. }
$$

and also the relations

$$
\begin{gathered}
\operatorname{Trace}[(\mathbf{A} \bullet \mathbf{B}) \bullet \mathbf{C}]=\operatorname{Trace}[\mathbf{A} \bullet(\mathbf{B} \bullet \mathbf{C})]=\operatorname{Trace}[\mathbf{A} \bullet \mathbf{B} \bullet \mathbf{C}]= \\
\operatorname{Trace}[\mathbf{C} \bullet \mathbf{A} \bullet \mathbf{B}]=\operatorname{Trace}[\mathbf{B} \bullet \mathbf{C} \bullet \mathbf{A}] .
\end{gathered}
$$

Taking into account these properties of the trace of Zorn matrix products, the novel line interval proposed in this work can be unambiguously defined by

$$
\begin{gathered}
d s^{2}=\frac{1}{4} \operatorname{Trace}\left[\mathbf{G}_{\mu \nu} \bullet d \mathbf{Z}^{\mu} \bullet d \mathbf{Z}^{\nu}\right]= \\
\frac{1}{2} g_{\mu \nu} d x_{o}^{\mu} d x_{o}^{\nu}+\frac{1}{2} s_{\mu \nu}^{k} d y_{k}^{\mu} d x_{o}^{\nu}+\frac{1}{2} g_{\mu \nu} d x_{k}^{\mu} d y_{k}^{\nu}+\frac{1}{2} s_{\mu \nu}^{k} d y_{k}^{\nu} d y_{o}^{\mu}+ \\
\frac{1}{2} \epsilon_{i j k} r_{\mu \nu}^{i} d y_{j}^{\mu} d y_{k}^{\nu}+\frac{1}{2} r_{\mu \nu}^{k} d x_{k}^{\nu} d x_{o}^{\mu}+\frac{1}{2} g_{\mu \nu} d y_{k}^{\mu} d x_{k}^{\nu}+ \\
\frac{1}{2} \epsilon_{i j k} s_{\mu \nu}^{i} d x_{j}^{\mu} d x_{k}^{\nu}+\frac{1}{2} r_{\mu \nu}^{k} d x_{k}^{\mu} d y_{o}^{\nu}+\frac{1}{2} g_{\mu \nu} d y_{o}^{\mu} d y_{o}^{\nu}
\end{gathered}
$$


It is very important to emphasize that the line interval considered by [20] did not involve octonionic valued spacetime coordinates, thus their line interval was given by :

$$
d s^{2}=\frac{1}{4} \operatorname{Trace}\left[\mathbf{G}_{\mu \nu} d x^{\mu} x^{\nu}\right]=\frac{1}{4}\left(\operatorname{Trace}\left[\mathbf{G}_{\mu \nu}\right]\right) d x^{\mu} x^{\nu}=g_{\mu \nu} d x^{\mu} d x^{\nu} .
$$

which clearly differs from the most general one when $x^{\mu} \rightarrow \mathbf{Z}^{\mu}$. The reason this choice of interval (22) was selected by [20] is because it is invariant under internal octonionic transformations acting on the metric and leaving invariant the ordinary spacetime coordinates $x^{\mu}$ since $d x^{\mu} d x^{\nu}=d x^{\mu} d x^{\nu} \mathbf{1}_{4 \times 4}$

$$
\mathbf{G}_{\mu \nu}^{\prime}=\mathbf{U} \bullet \mathbf{G}_{\mu \nu} \bullet \mathbf{U}^{-1}
$$

where

$$
\mathbf{U}=e^{\alpha_{i} u_{i}+\beta u_{i}^{*}} \equiv m_{o} u_{o}^{*}+n_{o} u_{o}+m_{i} u_{i}^{*}+n_{i} u_{i}
$$

such that

$$
\mathbf{Z}^{\prime \mu}=\frac{1}{2}\left[\left(\mathbf{U} \bullet \mathbf{Z}^{\mu}\right) \bullet \mathbf{U}^{-1}+\mathbf{U} \bullet\left(\mathbf{Z}^{\mu} \bullet \mathbf{U}^{-1}\right)\right] .
$$

If, and only if, $\mathbf{U}^{-1}=\overline{\mathbf{U}}$

$$
\mathbf{U}^{-1} \mathbf{U}=\mathbf{U U}^{-1}=\overline{\mathbf{U}} \mathbf{U}=\mathbf{U} \overline{\mathbf{U}}=\mathbf{1}_{4 \times 4}=\left(u_{o}+u_{o}^{*}\right) .
$$

then one can show $[20]$

$$
\mathbf{Z}^{\prime \mu}=\frac{1}{2}\left[\left(\mathbf{U} \bullet \mathbf{Z}^{\mu}\right) \bullet \mathbf{U}^{-1}+\mathbf{U} \bullet\left(\mathbf{Z}^{\mu} \bullet \mathbf{U}^{-1}\right)\right]=\mathbf{U} \bullet \mathbf{Z}^{\mu} \bullet \mathbf{U}^{-1}
$$

resulting from the Moufang identity $a(b \bar{a})=(a b) \bar{a}$ when $a=U$ and $b=Z^{\mu}$. Hence, using the cyclic properties of the trace of products of the Zorn matrices, it leads to

$$
\begin{gathered}
\text { Trace }\left[\mathbf{G}^{\prime}{ }_{\mu \nu}\right]= \\
\text { Trace }\left[\mathbf{U} \bullet \mathbf{G}_{\mu \nu} \bullet \mathbf{U}^{-1}\right]=\operatorname{Trace}\left[\mathbf{U}^{-1} \bullet \mathbf{U} \bullet \mathbf{G}_{\mu \nu}\right]= \\
\text { Trace }\left[\left(\mathbf{U}^{-1} \bullet \mathbf{U}\right) \bullet \mathbf{G}_{\mu \nu}\right]=\operatorname{Trace}\left[\mathbf{G}_{\mu \nu}\right]
\end{gathered}
$$

consequently, the line interval is $U$-invariant

$$
\frac{1}{4}\left(\operatorname{Trace}\left[\mathbf{G}^{\prime}{ }_{\mu \nu}\right]\right) d x^{\mu} d x^{\nu}=\frac{1}{4}\left(\operatorname{Trace}\left[\mathbf{G}_{\mu \nu}\right]\right) d x^{\mu} d x^{\nu} .
$$

However, when the spacetime coordinates are replaced by octononic valued ones, $x^{\mu} \rightarrow \mathbf{Z}^{\mu}$, it is not necessarily true that the transformation of the interval $(d s)^{2} \rightarrow\left(d s^{\prime}\right)^{2}$

$$
\left(d s^{\prime}\right)^{2}=\frac{1}{4} \operatorname{Trace}\left[\mathbf{G}_{\mu \nu}^{\prime} \bullet d \mathbf{Z}^{\prime \mu} \bullet d \mathbf{Z}^{\prime \nu}\right]=
$$




$$
\frac{1}{4} \operatorname{Trace}\left[\left(\mathbf{U} \bullet \mathbf{G}_{\mu \nu} \bullet \mathbf{U}^{-1}\right) \bullet\left(\mathbf{U} \bullet d \mathbf{Z}^{\mu} \bullet \mathbf{U}^{-1}\right) \bullet\left(\mathbf{U} \bullet d \mathbf{Z}^{\nu} \bullet \mathbf{U}^{-1}\right)\right] .
$$

remains invariant under the internal octonionic transformations. To prove this one may recur to the Moufang identity $(a b)(c a)=a(b c) a$ in the simpler case when $\mathbf{G}_{\mu \nu}$ is reduced to an ordinary Minkowski (diagonal) metric $\eta_{\mu \nu} \mathbf{1}_{4 \times 4}$ :

$\frac{1}{4} \eta_{\mu \nu} \operatorname{Trace}\left[d \mathbf{Z}^{\prime \mu} \bullet d \mathbf{Z}^{\prime \nu}\right]=\frac{1}{4} \eta_{\mu \nu} \operatorname{Trace}\left[\left(\mathbf{U} \bullet d \mathbf{Z}^{\mu} \bullet \mathbf{U}^{-1}\right) \bullet\left(\mathbf{U} \bullet d \mathbf{Z}^{\nu} \bullet \mathbf{U}^{-1}\right)\right]$

By setting

$$
\begin{gathered}
U d Z=a, U^{-1}=b, U=c, d Z U^{-1}=d \neq a \Rightarrow(a b)(c d) \neq a(b c) d \Rightarrow \\
\left(U d Z U^{-1}\right)\left(U d Z U^{-1}\right) \neq U d Z\left(U^{-1} U\right) d Z U^{-1}=(U d Z)\left(d Z U^{-1}\right) \Rightarrow \\
\frac{1}{4} \eta_{\mu \nu} \operatorname{Trace}\left[\left(\mathbf{U} \bullet d \mathbf{Z}^{\mu} \bullet \mathbf{U}^{-1}\right) \bullet\left(\mathbf{U} \bullet d \mathbf{Z}^{\nu} \bullet \mathbf{U}^{-1}\right)\right] \neq \\
\frac{1}{4} \eta_{\mu \nu} \operatorname{Trace}\left[\left(\mathbf{U} \bullet d \mathbf{Z}^{\mu}\right) \bullet\left(d \mathbf{Z}^{\nu} \bullet \mathbf{U}^{-1}\right)\right]= \\
\frac{1}{4} \eta_{\mu \nu} \operatorname{Trace}\left[\left(d \mathbf{Z}^{\nu} \bullet \mathbf{U}^{-1}\right) \bullet\left(\mathbf{U} \bullet d \mathbf{Z}^{\mu}\right)\right]= \\
\frac{1}{4} \eta_{\mu \nu} \operatorname{Trace}\left[d \mathbf{Z}^{\nu} \bullet\left(\mathbf{U}^{-1} \bullet \mathbf{U}\right) \bullet d \mathbf{Z}^{\mu}\right]= \\
\frac{1}{4} \eta_{\nu \mu} \operatorname{Trace}\left[\left(d \mathbf{Z}^{\nu} \bullet d \mathbf{Z}^{\mu}\right]=\frac{1}{4} \eta_{\mu \nu} \operatorname{Trace}\left[\left(d \mathbf{Z}^{\mu} \bullet d \mathbf{Z}^{\nu}\right] .\right.\right.
\end{gathered}
$$

due to the symmetry of $\eta_{\mu \nu}=\eta_{\nu \mu}$ of the ordinary Minkowski (diagonal) metric.

Therefore, one can see by means of the Moufang identities and the cyclic trace property why the interval ( 14 ) is not invariant under $U$-transformations because the transformed interval of eq-(30) is not the same as the original interval of eq-(14). The same conclusion applies to the most general interval involving octonionic valued metric and coordinates. Nevertheless, there is invariance with respect to a $S U(2)$ diagonal subalgebra of $S U(2)_{L} \times S U(2)_{R}$ in the restricted case that

$$
\begin{gathered}
m_{o}=n_{o}, m_{i}=n_{i} \Rightarrow \mathbf{U}=e^{\alpha_{i} u_{i}+\beta u_{i}^{*}} \equiv m_{o}\left(u_{o}^{*}+u_{o}\right)+m_{i}\left(u_{i}^{*}+u_{i}\right)= \\
m_{o} \omega_{o}+m_{i} \omega_{i}
\end{gathered}
$$

and it reduces to the standard associative quaternionic $U$ transformation. In this restricted quaternionic case the line interval (29) would remain invariant when $\mathbf{U}$ is quaternionc valued becuase quaternions are associative. Thus using the associative property of quaternionic products, and the cyclic trace property, leads to an $U$-invariant line interval if, and only if, the $\mathbf{G}_{\mu \nu}$ and $\mathbf{Z}^{\mu}$ are also restricted to be quaternionic-valued, meaning that now one must impose the 
constraints $x_{o}^{\mu}=y_{o}^{\mu}$ and $x_{i}^{\mu}=y_{i}^{\mu}$ among the components of the split-octonion $\mathbf{Z}^{\mu}$, and $\mathcal{F}_{\mu \nu}^{(1)}=\mathcal{F}_{\mu \nu}^{(2)}$ among the components of the metric.

Therefore, under these conditions, the line interval (and bilinear forms) given by eq-(21) are invariant under the diagonal subalgebra $S U(2)_{\text {diag }} \subset S U(2)_{L} \times$ $S U(2)_{R}$; i.e. under internal quaternionic $U$-rotations the interval $d s^{2}$ remains invariant. In the case of ordinary spacetime coordinates $x^{\mu}$ but octonionic valued metric $\mathbf{G}_{\mu \nu}$ the octonionc affinity is [20]

$$
\boldsymbol{\Upsilon}_{\mu \nu}^{\rho}=\Omega_{\mu \nu}^{\rho}\left(u_{o}+u_{o}^{*}\right)+\delta_{\mu}^{\rho}\left[\mathbf{L}_{\nu i} u_{i}^{*}+\mathbf{K}_{\nu_{i}} u_{i}\right] .
$$

where the spacetime connection is

$$
\Omega_{\mu \nu}^{\rho}=\Gamma_{(\mu \nu)}^{\rho}+i \Gamma_{[\mu \nu]}^{\rho} .
$$

naturally there is a nontrivial torsion due to the antisymmetric components of the connection.

The octonionic curvature is :

$$
\mathbf{R}_{\rho_{\mu \nu}}^{\sigma}=\mathcal{R}_{\rho \mu \nu}^{\sigma}\left[\Omega_{\mu \nu}^{\rho}\right]\left(u_{o}+u_{o}^{*}\right)+\delta_{\rho}^{\sigma} \mathbf{P}_{\mu \nu} .
$$

where $\mathcal{R}_{\rho \mu \nu}^{\sigma}\left[\Omega_{\mu \nu}^{\rho}\right]$ is the ordinary spacetime curvature corresponding to the connection $\Omega_{\mu \nu}^{\rho}$. The internal space curvature is given by

$$
\mathbf{P}_{\mu \nu}=\boldsymbol{\Theta}_{\mu, \nu}-\boldsymbol{\Theta}_{\nu, \mu}-\left[\boldsymbol{\Theta}_{\mu}, \boldsymbol{\Theta}_{\nu}\right] .
$$

in terms of the internal space affinity

$$
\boldsymbol{\Theta}_{\mu}=\left[\mathbf{L}_{\nu i} u_{i}^{*}+\mathbf{K}_{\nu_{i}} u_{i}\right] .
$$

The internal space covariant derivative of an octonionic-valued scalar field $\boldsymbol{\Phi}\left(x^{\mu}\right)$ is

$$
D_{\mu} \mathbf{\Phi}=\mathbf{\Phi}_{, \mu}+\left[\boldsymbol{\Theta}_{\mu}, \boldsymbol{\Phi}\right] .
$$

The commutator of two internal space covariant derivatives

$$
\left[D_{\mu}, D_{\nu}\right] \boldsymbol{\Phi}=\mathbf{P}_{\mu \nu} \boldsymbol{\Phi}-\boldsymbol{\Phi} \mathbf{P}_{\nu \mu}+\left\{\boldsymbol{\Theta}_{\mu}, \boldsymbol{\Theta}_{\nu}, \boldsymbol{\Phi}\right\} .
$$

contains a crucial non-vanishing associator term $\left\{\boldsymbol{\Theta}_{\mu}, \boldsymbol{\Theta}_{\nu}, \boldsymbol{\Phi}\right\}$ which is the hallmark of the octonionic structure; also there is a nontrivial ordering of the terms $\mathbf{P}_{\mu \nu} \mathbf{\Phi}-\mathbf{\Phi} \mathbf{P}_{\nu \mu}$ that is a reflection of the noncommutativity.

The full covariant derivative (from the spacetime and internal space perspective) of a split-octonionic valued spacetime tensor is

$$
\mathcal{D}_{\alpha} \mathbf{A}_{\mu}^{\rho}=\mathbf{A}_{\mu, \alpha}^{\rho}+\Omega_{\sigma \alpha}^{\rho} \mathbf{A}_{\mu}^{\sigma}-\Omega_{\mu \alpha}^{\sigma} \mathbf{A}_{\sigma}^{\rho}+\left[\mathbf{\Theta}_{\alpha}, \mathbf{A}_{\mu}^{\rho}\right] .
$$

the commutator of two derivatives is

$$
\left[\mathcal{D}_{\alpha}, \mathcal{D}_{\beta}\right] \mathbf{A}^{\mu}=\mathbf{R}_{\rho \alpha \beta}^{\mu} \mathbf{A}^{\rho}+\mathbf{A}^{\mu} \mathbf{P}_{\alpha \beta}+\left\{\Upsilon_{\rho \alpha}^{\mu}, \Upsilon_{\tau \beta}^{\rho}, \mathbf{A}^{\tau}\right\}-
$$




$$
\left\{\boldsymbol{\Upsilon}_{\rho \beta}^{\mu}, \mathbf{\Upsilon}_{\tau \alpha}^{\rho}, \mathbf{A}^{\tau}\right\}+4\left\{\boldsymbol{\Theta}_{\alpha}, \boldsymbol{\Theta}_{\beta}, \mathbf{A}^{\mu}\right\}-2 \mathbf{A}_{; \rho}^{\mu} \Omega_{[\alpha \beta]}^{\rho} .
$$

where the full-fledged curvature tensor in eq-(35) involving the spacetime and internal space curvature can also be written as

$$
\mathbf{R}_{\rho \alpha \beta}^{\mu}=\mathbf{\Upsilon}_{\rho \alpha, \beta}^{\mu}-\mathbf{\Upsilon}_{\rho \beta, \alpha}^{\mu}+\mathbf{\Upsilon}_{\tau \beta}^{\mu} \mathbf{\Upsilon}_{\rho \alpha}^{\tau}-\mathbf{\Upsilon}_{\tau \alpha}^{\mu} \mathbf{\Upsilon}_{\rho \beta}^{\tau}
$$

the spacetime covariant derivative is

$$
\mathbf{A}_{; \alpha}^{\mu}=\mathbf{A}_{, \alpha}^{\mu}+\Omega_{\rho \alpha}^{\mu} \mathbf{A}^{\rho} .
$$

and the Ricci tensor is $\mathbf{R}_{\mu \nu}=\mathbf{R}_{\mu \rho \nu}^{\rho}$. The inverse $\mathbf{G}^{\mu \nu}$ is such $\mathbf{G}^{\mu \nu} \mathbf{G}_{\mu \nu}=$ $\delta_{\mu}^{\nu}\left(u_{o}+u_{o}^{*}\right)$ which permits to construct the scalar curvature $\mathbf{R}=\mathbf{G}^{\mu \nu} \mathbf{R}_{\mu \nu}$ and the analog of the Einstein-Hilbert action [20]. A Palatini independent variation of the metric and connection furnishes the equations of motion of the coupled Einstein-Maxwell-Yang-Mills equations

$$
\begin{gathered}
\mathbf{R}_{\mu \nu}=0, \quad \frac{1}{\sqrt{|G|}} \partial_{\nu}\left(\sqrt{|G|} F^{\mu \nu}\right)=0 . \\
-\frac{1}{\sqrt{|G|}} \partial_{\nu}\left(\sqrt{|G|} \mathcal{F}_{(1) k}^{\mu \nu}\right)+2 \epsilon_{i j k} \mathbf{K}_{\nu i} \mathcal{F}_{(2) j}^{\mu \nu}=0 \\
-\frac{1}{\sqrt{|G|}} \partial_{\nu}\left(\sqrt{|G|} \mathcal{F}_{(2) k}^{\mu \nu}\right)+2 \epsilon_{i j k} \mathbf{L}_{\nu i} \mathcal{F}_{(1) j}^{\mu \nu}=0 .
\end{gathered}
$$

where $G=\frac{1}{4}$ Trace $\left[\operatorname{det}\left(\mathbf{G}_{\mu \nu}\right)\right]$.

The construction of the full-fledged octonionic gravity complicates even further when the spacetime coordinates themselves are octonionic valued, $x^{\mu} \rightarrow \mathbf{Z}^{\mu}$, since now the left and right derivatives are not the same; the line interval is not $U$-invariant as we have shown, it is only invariant under the $S U(2)_{\text {diag }} \subset$ $S U(2)_{L} \times S U(2)_{R}$ when restrictions are imposed; among other subtleties dealing with the notion of octonionic/quaternionic analytical functions. As far as we know this nonassociative and noncommutative octonionic spacetime geometry has not been developed so far. It is warranted to develop the full fledged nonassociative and noncommutative octonionic gravity because it is very appealing for the reasons that it would not require to introduce Quantum Groups, Fuzzy spaces, Moyal and Hopf algebraic deformations of the spacetime Poincare algebra; nor to introduce by hand the noncommutativity of the spacetime coordinates and momenta [54], [55], [53].

The split-octonionic momentum is

$$
\mathbf{P}^{\mu}=p_{o}^{\mu} u_{o}^{*}+q_{o}^{\mu} u_{o}+\pi_{i}^{\mu} u_{i}^{*}+\sigma_{i}^{\mu} u_{i}=\left(\begin{array}{ccc}
p_{o}^{\mu} & \omega_{o} & -\pi_{i}^{\mu} \omega^{i} \\
\sigma_{i}^{\mu} & \omega^{i} & q_{o}^{\mu} \omega_{o}
\end{array}\right)
$$

An immediate consequence of the noncommutative and nonassociative spacetime geometry is the modified dispersion relations which can be inferred from the expression of the norm of an octonionic-valued momentum vector $\mathbf{P}^{\mu}$ 


$$
\begin{gathered}
\left\|\mathbf{G}_{\mu \nu} \mathbf{P}^{\mu} \mathbf{P}^{\nu}\right\|=\frac{1}{4} \operatorname{Trace}\left[\mathbf{G}_{\mu \nu} \bullet \mathbf{P}^{\mu} \bullet \mathbf{P}^{\nu}\right]= \\
\frac{1}{2} g_{\mu \nu} p_{o}^{\mu} p_{o}^{\nu}+\frac{1}{2} s_{\mu \nu}^{k} \sigma_{k}^{\mu} p_{o}^{\nu}+\frac{1}{2} g_{\mu \nu} \pi_{k}^{\mu} \sigma_{k}^{\nu}+\frac{1}{2} s_{\mu \nu}^{k} \sigma_{k}^{\nu} q_{o}^{\mu}+ \\
\frac{1}{2} \epsilon_{i j k} r_{\mu \nu}^{i} \sigma_{j}^{\mu} \sigma_{k}^{\nu}+\frac{1}{2} r_{\mu \nu}^{k} \pi_{k}^{\nu} p_{o}^{\mu}+\frac{1}{2} g_{\mu \nu} \sigma_{k}^{\mu} \pi_{k}^{\nu}+ \\
\frac{1}{2} \epsilon_{i j k} s_{\mu \nu}^{i} \pi_{j}^{\mu} \pi_{k}^{\nu}+\frac{1}{2} r_{\mu \nu}^{k} \pi_{k}^{\mu} q_{o}^{\nu}+\frac{1}{2} g_{\mu \nu} q_{o}^{\mu} q_{o}^{\nu}
\end{gathered}
$$

When one imposes the constraints $p_{o}^{\mu}=q_{o}^{\mu}$ and $\pi_{i}^{\mu}=\sigma_{i}^{\mu}$ among the components of the split-octonion $\mathbf{P}^{\mu}$, and $\mathcal{F}_{\mu \nu}^{(1)}=\mathcal{F}_{\mu \nu}^{(2)}$ among the components of the metric, then the norm $\left\|\mathbf{G}_{\mu \nu} \mathbf{P}^{\mu} \mathbf{P}^{\nu}\right\|$ given by eq-(47) is invariant under the diagonal subalgebra $S U(2)_{d i a g} \subset S U(2)_{L} \times S U(2)_{R}$; i.e. under the internal quaternionic $U$-rotations, the momentum norm (47) remains invariant.

Under these restrictions on the components, the corrections of order $\kappa^{2}$ to the ordinary norm

$$
\frac{1}{2} g_{\mu \nu}\left(p_{o}^{\mu} p_{o}^{\nu}+q_{o}^{\mu} q_{o}^{\nu}+2 \pi_{k}^{\mu} \sigma_{k}^{\nu}\right)=g_{\mu \nu}\left(p_{o}^{\mu} p_{o}^{\nu}+\pi_{k}^{\mu} \pi_{k}^{\nu}\right) .
$$

are then given by

$$
(\kappa)^{2}(\lambda)^{2} \epsilon_{i j k} \mathcal{F}_{\mu \nu}^{i} \pi_{j}^{\mu} \pi_{k}^{\nu}+\ldots=(\kappa)^{2}(\lambda)^{2} \overrightarrow{\mathcal{F}}_{\mu \nu} . \vec{\Sigma}^{\mu \nu}+\ldots \ldots
$$

One of the most important novel conclusions of this work is that one has found in eq- (47), (49) the modified dispersion relations of the momentum, whose corrections are proportional to the length scale squared $\kappa^{2}$ (of the order of the Planck scale squared, i.e. $1 / M_{\text {Planck }}^{2}$ ) involving the coupling of the internal components of the left/right isospin-momentum $\pi_{k}^{\mu}, \sigma_{k}^{\mu}$ to the $S U(2)_{L}, S U(2)_{R}$ Yang-Mills field strengths $\mathcal{F}_{\mu \nu}^{(1)}, \mathcal{F}_{\mu \nu}^{(2)}$. In the particular case that

$$
s_{\mu \nu}^{k} \sigma_{k}^{\mu}=r_{\mu \nu}^{k} \pi_{k}^{\nu}=0
$$

the $O\left(\kappa^{2}\right) \sim O\left(1 / M_{\text {Planck }}^{2}\right)$ corrections are due entirely to the flux of the YangMills fields through the area-momentum in the internal isospin space

$$
\Sigma_{i}^{\mu \nu}=\epsilon_{i j k} \pi_{j}^{\mu} \pi_{k}^{\nu}
$$

given by

$$
\Phi_{\text {flux }}=\epsilon_{i j k} \mathcal{F}_{\mu \nu}^{i} \pi_{j}^{\mu} \pi_{k}^{\nu}=\overrightarrow{\mathcal{F}}_{\mu \nu} \cdot \vec{\Sigma}^{\mu \nu},
$$

Thus, the flux of $\overrightarrow{\mathcal{F}}_{\mu \nu}$ through the area-momentum $\vec{\Sigma}^{\mu \nu}$ in the internal isospin space yields the corrections $O\left(1 / M_{\text {Planck }}^{2}\right)$ to the energy-momentum dispersion relations and without violating Lorentz invariance.

This finding is not unique to octonions and quaternions. Next we will review how the minimal length string uncertainty relations can be obtained from 
polyparticle dynamics in Clifford-spaces (C-spaces) [50]. The truly C-space invariant norm of a momentum poly-vector is defined (after introducing suitable powers of the Planck mass that is set to unity in order to match units)

$$
\|P\|^{2}=\pi^{2}+p_{\mu} p^{\mu}+p_{\mu \nu} p^{\mu \nu}+p_{\mu \nu \rho} p^{\mu \nu \rho}+\ldots=M^{2}
$$

The way to generate different expressions for the $M^{2}$ is by taking slices (sections ) of the $2^{D}$-dim mass-shell hyper-surface in C-space onto subspaces of smaller dimensionality. This is achieved by imposing the following constraints on the components of the poly-vector-momentum. In doing so one is explicitly breaking the poly-dimensional covariance and for this reason one can obtain an infinity of possible choices for the Casimirs $M^{2}$.

To demonstrate this, we impose the following constraints :

$$
p_{\mu \nu} p^{\mu \nu}=a_{2}\left(p_{\mu} p^{\mu}\right)^{2}=a_{2} p^{4} . \quad p_{\mu \nu \rho} p^{\mu \nu \rho}=a_{3}\left(p_{\mu} p^{\mu}\right)^{3}=a_{3} p^{6} .
$$

Upon doing so the norm of the poly-momentum becomes:

$$
\|P\|^{2}=P_{A} P^{A}=\sum_{n=0}^{n=D} a_{n} p^{2 n}=M^{2}\left(a_{o}, a_{2}, a_{3}, \ldots, a_{D}\right)
$$

Therefore, by a judicious choice of the coefficients $a_{n}$, and by reinserting the suitable powers of the Planck scale, which have to be there in order to combine objects of different dimensions, one can reproduce all the possible Casimirs in the form:

$$
M^{2}=m^{2}[f(\Lambda m / \hbar)]^{2} . \quad m^{2} \equiv p_{\mu} p^{\mu}=p^{2} .
$$

The simplest way to infer the effects of the poly-vector-valued coordinates of C-space on the commutation relations is by working with an effective energy dependent $\hbar_{e f f}$ that appears in the nonlinear de Broglie dispersion relation. The mass-shell condition in C-space, after imposing the constraints among the poly-vector valued components, yields an effective mass $M=m f(\Lambda m / \hbar)$. The generalized de Broglie relations, which are no longer linear, are [50]

$$
\begin{gathered}
\left|P_{\text {effective }}\right|=|p| f(\Lambda m / \hbar)=\hbar_{\text {effective }}\left(k^{2}\right)|k| \\
\hbar_{\text {effective }}\left(k^{2}\right)=\hbar f(\Lambda m / \hbar)=\hbar \sum_{n=0}^{n=N} a_{n}(\Lambda m / \hbar)^{2 n}=\hbar \sum_{n=0}^{n=N} a_{n}(\Lambda k)^{2 n} \\
m^{2}=p^{2}=p_{\mu} p^{\mu}=(\hbar k)^{2} .
\end{gathered}
$$

where the upper limit in the sum $N=D$ is given by the spacetime dimension. Using the effective $\hbar_{e f f}$ in the well known relation based on the Schwartz inequality and the fact that $|z| \geq|\operatorname{Im} z|$ leads to

$$
\Delta x^{i} \Delta p^{j} \geq \frac{1}{2}\left\|<\left[x^{i}, p^{j}\right]>\right\| . \quad\left[x^{i}, p^{j}\right]=i \hbar_{e f f}\left(k^{2}\right) \delta^{i j} .
$$


The use of the inequalities,

$$
<p^{2}>\geq(\Delta p)^{2} ;<p^{4}>\geq(\Delta p)^{4} ; \ldots \ldots<p^{2 n}>\geq(\Delta p)^{2 n}
$$

in the above equations leads to :

$$
\begin{aligned}
<\hbar_{\text {effective }}\left(k^{2}\right)> & \geq \hbar \sum_{n=0}^{n=N} a_{n}(\Lambda / \hbar)^{2 n}\left(<\left(p_{n}\right)^{2 n}>\right) \geq \\
& \hbar \sum_{n=0}^{n=N} a_{n}(\Lambda / \hbar)^{2 n}(\Delta p)^{2 n} .
\end{aligned}
$$

Finally, we get that for each pair of conjugate canonical variables $\left(x, p_{x}\right) ;\left(y, p_{y}\right) ;\left(z, p_{z}\right) ; .$. the product of uncertainties (we omit indices for simplicity) is given by

$$
\Delta x \Delta p \geq \frac{1}{2} \hbar+\frac{a_{1} \hbar}{2}\left(\frac{\Lambda}{\hbar}\right)^{2}(\Delta p)^{2}+\frac{a_{2} \hbar}{2}\left(\frac{\Lambda}{\hbar}\right)^{4}(\Delta p)^{4}+\ldots \ldots \ldots \ldots
$$

The second term of last relation yields the stringy contribution to the modified uncertainty relations, whereas the higher order corrections in eq-(58) stem from the higher rank components of the poly-momentum and represent the membrane, 3 -brane.... and $D-1$-brane contributions to the generalized uncertainty relations given by :

$$
\Delta x \geq \frac{\hbar}{2 \Delta p}+\frac{a_{1}}{2} \frac{\Lambda^{2}}{\hbar} \Delta p+\frac{a_{2}}{2} \frac{\Lambda^{4}}{\hbar^{3}}(\Delta p)^{3}+\ldots .
$$

By replacing lengths by times and momenta by energy one reproduces the minimal Planck time uncertainty relations. By keeping only the first two terms of eq- $(60)$ one can infer that there is a minimum uncertainty of the order of the Planck scale $\Lambda$.

QM in Clifford-spaces (C-spaces) is very rich with many novelties [50]. A novel Weyl-Heisenberg algebra in Clifford-spaces was constructed that is based on a matrix-valued $\mathcal{H}^{A B}$ extension of Planck's constant [50]. As a result of this modified Weyl-Heisenberg algebra one will no longer be able to measure, simultaneously, the pairs of variables $\left(x, p_{x}\right) ;\left(x, p_{y}\right) ;\left(x, p_{z}\right) ;\left(y, p_{x}\right), \ldots$ with absolute precision. New Klein-Gordon and Dirac wave equations and dispersion relations in Clifford-spaces were presented. The latter Dirac equation were a generalization of the Dirac-Lanczos-Barut-Hestenes equation.

Another important conclusion deals with the role of the anti-symmetric component of the metric $g_{[\mu \nu]}=\kappa^{2} F_{\mu \nu}$ that is related to the Maxwell EM field strength in the Einstein-Strauss, Moffat-Boal nonsymmetric theory of gravity [19]. The Noncommutative momentum variables in Yang's noncommutative phase space spacetime obey the algebra 


$$
\left[p^{\mu}, p^{\nu}\right]=i\left(\frac{\hbar}{R}\right)^{2} J^{\mu \nu}
$$

where $J^{\mu \nu}$ is the generator of the Lorentz algebra (angular momentum) and $R$ is an infrared upper cutoff length scale ( a minimum momentum). Consequently, the norm of the Moyal-Kontsevich star product

$$
\left[g_{(\mu \nu)}+i g_{[\mu \nu]}\right] p^{\mu} * p^{\nu}=g_{(\mu \nu)} p^{\mu} p^{\nu}-\left(\frac{\hbar}{R}\right)^{2} g_{[\mu \nu]} J^{\mu \nu}+\ldots
$$

will receive corrections of the order $\left(\frac{\hbar}{R}\right)^{2} \kappa^{2} F_{\mu \nu} J^{\mu \nu}$.

An explicit isomorphism between Yang's Noncommutative space-time algebra and the area-coordinates algebra associated with Clifford spaces was found in [50]. The former Yang's algebra involves noncommuting coordinates and momenta with a minimum Planck scale $\Lambda$ (ultraviolet cutoff) and a minimum momentum $p=\hbar / R$ (maximal length $R$, infrared cutoff ). The double-scaling limit of Yang's algebra $\Lambda \rightarrow 0, R \rightarrow \infty$, in conjunction with the large $n \rightarrow \infty$ limit, leads naturally to the area quantization condition $\Lambda R=L^{2}=n \Lambda^{2}$ ( in Planck area units ) given in terms of the discrete angular-momentum eigenvalues $n$. Modified Newtonian dynamics were also a consequence of Yang's algebra resulting from the modified Poisson brackets.

To finalize, we must add that Nonassociative Gauge theories based on the Moufang $S^{7}$ loop product ( not a Lie algebra ) have been constructed by [39]. They are based on the Moufang $S^{7}$-loop valued field strength

$$
F=d A+A \wedge A+g^{-1}\{g, A, A\} .
$$

where $g$ is an $S^{7}$-valued function, called a gauge, it is a section of the $S^{7}$ principal bundle and physically it represents a frame with respect to which one can describe charged fields. Taking the algebra of octonions with a unit norm as the Moufang $S^{7}$-loop one reproduces a nonassociative octonionic gauge theory which is a generalization of the Maxwell and Yang-Mills gauge theories based on Lie algebras. BPST-like instantons solutions in $D=8$ were also found. These solutions represented the physical degrees of freedom of the transverse 8-dimensions of superstring solitons in $D=10$ preserving one and two of the 16 spacetime supersymmetries.

Nonassociative Gauge theories based on the left and right bimodules of the octonionic algebra were constructed by [38]. The octonionic gauge formulation of EM Dyons involving both electric $e$ and magnetic charges $g$ (which couple to a complex-valued potential $V_{\mu}=A_{\mu}-i B_{\mu}$ ) and Gravitational-Dyons involving real and imaginary masses have been studied by [37], [38].

An $E_{8}$ exceptional geometric structure of $D=11$ Supergravity was conjectured by [59] after performing a $3+8$ split of the fields in $D=11$. The exceptional metric $G_{M N}$ whose indices $M, N$ involve 8 vector $m=1,2, \ldots 8$ indices and 28 bi-vector-valued indices $[\mathrm{mn}]$ in $d=8$-dimensions. The metric $G_{M N}$ encoded the propagating bosonic fields $g_{m n}, A_{[m n p]}$ of Supergravity in one scoop and was explicitly given in terms of a generalized $248 \times 36$ matrix $\mathcal{V}_{M}^{\mathcal{A}}$ by 


$$
G_{M N}=\mathcal{V}_{M}^{\mathcal{A}} \mathcal{V}_{N}^{\mathcal{A}}, \quad M=m,[m n]=8+28=36 ; \quad \mathcal{A}=1,2,3, \ldots \ldots, 248 .
$$

the $\mathcal{A}$ indices run over the 248 dimenions of $E_{8}$.

Despite that octonions are nonassociative there are in fact Octonionic realizations of the (associative) $C l(8)$ and $C l(4)$ algebras in [46], [45], [38], by means of left/right products. The known Octonionic realizations of the Clifford $\mathrm{Cl}(8), C l(4)$ algebras should permit the construction of octonionic string actions that should have a correspondence with ordinary string actions for strings moving in a curved Clifford-space target background associated with a $C l(3,1)$ algebra,. For instance, the $C l(3,1)$-space interval involving poly-vectors admits an $8+8$ split [22] of the form

$$
\begin{gathered}
|d \mathbf{X}|^{2}=d \Omega^{2}+\left(d x^{0}\right)^{2}-\left(d x^{i}\right)^{2}-\left(d x^{0 i}\right)^{2}+\left(d x^{i j}\right)^{2}-\left(d x^{123}\right)^{2}+ \\
\left(d x^{023}\right)^{2}+\left(d x^{013}\right)^{2}+\left(d x^{012}\right)^{2}-\left(d x^{0123}\right)^{2} ; \quad i, j, k=1,2,3 .
\end{gathered}
$$

when a Clifford-valued poly-vector in $D=4$ ( after setting the Planck scale to unity ) admits the expansion

$$
\mathbf{X}=\mathbf{X}^{M} E_{M}=\Omega \mathbf{1}+x^{\mu} \gamma_{\mu}+x^{[\mu \nu]} \gamma_{[\mu \nu]}+x^{[\mu \nu \rho]} \gamma_{[\mu \nu \rho]}+x^{[\mu \nu \rho \tau]} \gamma_{[\mu \nu \rho \tau]} .
$$

and can be decomposed into a scalar, vector, second rank antisymmetric tensor, axial-vector and pseudo-scalar yielding a total of $2^{4}=16=8+8$ components.

An octonionic string ( p-brane ) action is defined in terms of octonionicvalued maps $\mathbf{Z}^{\mu}\left(\xi^{a}\right), \quad a=1,2, \ldots, p+1$ from the $p+1$-dim world-volume of the string ( p-brane ) into the octonionic-valued target spacetime background $\mathbf{Z}^{\mu}, \mu=1,2,3, \ldots . . D$, as

$$
S=\frac{T_{p}}{2} \int d^{p+1} \xi \sqrt{h} h^{a b}\left[\frac{1}{4} \operatorname{Trace}\left(\partial_{a} \mathbf{Z}^{\mu} \bullet \partial_{b} \mathbf{Z}^{\nu} \bullet \mathbf{G}_{\mu \nu}\right)-(p-1)\right] .
$$

where $h^{a b}$ is the auxiliary $p+1$-dim world-volume metric correspoding to the $p$-brane. When $p=1$ the string world-sheet is two-dim, thus the embedding spacetime target background must have at least $D=2$ and whose two octonionic-valued spacetime coordinates are $\mathbf{Z}^{\mu}=\mathbf{Z}^{0}, \mathbf{Z}^{1}$ with a net number of $2 \times 8=16$ real components that match the number of $2^{4}=16$ components of a Clifford poly-vector $\mathbf{X}$ corresponding to the Clifford algebra $C l(3,1)$. We leave for future investigation the correspondence between the octonionc string action (68) $(p=1)$ and an ordinary string action corresponding to a string moving in a curved Clifford space background given by [21]

$$
S=\frac{T}{2} \int d^{2} \xi \sqrt{h} h^{a b} \partial_{a} \mathbf{X}^{M} \partial_{b} \mathbf{X}^{N} \mathbf{G}_{M N} .
$$

where $\mathbf{G}_{M N}$ is the curved C-space ( Clifford space ) metric that can be decomposed into sums of antisymmetrized products of the ordinary $D=4$ spacetime metric as 


$$
\begin{gathered}
\mathbf{G}_{\mu \nu}=g_{\mu \nu}, \quad \mathbf{G}_{\mu_{i_{1}} \mu_{i_{2}} \nu_{j_{1}} \nu_{j_{2}}}=\frac{1}{2 !} \epsilon_{i_{1} i_{2}} \epsilon_{j_{1} j_{2}} g_{\mu_{i_{1}} \nu_{j_{1}}} g_{\mu_{i_{2}} \nu_{j_{2}}} . \\
\mathbf{G}_{\mu_{i_{1}} \mu_{i_{2}} \mu_{i_{3}} \nu_{j_{1}} \nu_{j_{2}} \nu_{j_{3}}}=\frac{1}{3 !} \epsilon_{i_{1} i_{2} i_{3}} \epsilon_{j_{1} j_{2} j_{3}} g_{\mu_{i_{1}} \nu_{j_{1}}} g_{\mu_{i_{2}} \nu_{j_{2}}} g_{\mu_{i_{3}} \nu_{j_{3}}} . \\
\mathbf{G}_{\mu_{i_{1}} \mu_{i_{2}} \mu_{i_{3}} \mu_{i_{4}} \nu_{j_{1}} \nu_{j_{2}} \nu_{j_{3}} \nu_{j_{4}}}=\frac{1}{4 !} \epsilon_{i_{1} i_{2} i_{3} i_{4}} \epsilon_{j_{1} j_{2} j_{3} j_{4}} g_{\mu_{i_{1}} \nu_{j_{1}}} g_{\mu_{i_{2}} \nu_{j_{2}}} g_{\mu_{i_{3}} \nu_{j_{3}}} g_{\mu_{i_{4}} \nu_{j_{4}}} .
\end{gathered}
$$

A lot remains ahead, some preliminary work can be found in [47], [48], [49], $[50],[51],[52],[53],[12]$.

\section{Acknowledgements}

We are indebted to M. Bowers for invaluable assistance in preparing the manuscript. Special thanks to T. Smith for numerous discussions of his work.

\section{References}

[1] P. Jordan, J von Neumann and E. Wigner, Ann. Math 35 ( 1934 ) 2964. K. MacCrimmon, "A Taste of Jordan Algebras" H. Freudenthal, Nederl. Akad. Wetensch. Proc. Ser 57 A (1954 ) 218. J. Tits, Nederl. Akad. Wetensch. Proc. Ser 65 A (1962 ) 530. T. Springer, Nederl. Akad. Wetensch. Proc. Ser 65 A (1962) 259.

[2] J. Adams, " Lectures on Exceptional Lie Groups" Chicago Lectures in Mathematics, Univ. of Chicago Press 1996.

[3] R. Schafer, " An introduction to Nonassociative Algebras" (Academic Press 1966).

[4] C. H Tze and F. Gursey, "On the role of Divison, Jordan and Related Algebras in Particle Physics" World Scientific 1996. S. Okubo, Introduction to Octonion and other Nonassociative Algebras in Physics" Cambridge Univ. Press .

[5] R. Kerner, "Ternary Algebraic Structures and their applications in Physics" Proceedings of the Conference ICGTMP "Group-23", Dubna, Russia, July 30 - August 6, 2000[arXiv : math-ph/0011023] . Communications in Math. Phys. 91 (1983) 213; Classical and Quantum Gravity 14 (1A) (1997) 203.

[6] M. Dubois-Violette, R. Kerner and J. Madore, Jour Math Phys 31 (1990) 316; ibid (1990) 323. M. Dubois-Violette and M.Henneaux, Lett. Math. Phys. 49 (1999) 245. M. Dubois-Violette and M.Henneaux, Communications in Math. Phys. 226 (2002) 393; 
[7] N. Bazunova, A. Borowiec and R. Kerner, "Universal differential calculus on ternary algebras" Lett. Math. Phys. 67: 195-206, 2004 [ arXiv:math$\mathrm{ph} / 0401018$ ].

[8] T. Springer and F. Veldkamp, " Octonions, Jordan Algebras and Exceptional Groups" (Springer Verlag 2000)

[9] G. Dixon, " Division Algebras, Octonions, Quaternions, Complex Numbers, and the Algebraic Design of Physics" ( Kluwer, Dordrecht, 1994). J. Math. Phys 45, no 10 (2004) 3678.

[10] A. Einstein, Ann. Math 46 (1945) 578. Rev. Mod. Phys 20 (1948) 35. A. Einstein and E. Strauss, Ann. Math 47 (1946) 731.

[11] Y. Ohwashi, " $E_{6}$ Matrix Model " hep-th/0110106 $S p(4, H) / Z_{2}$ Pair Universe in $E_{6}$ Matrix Models" hep-th/0510252.

[12] C. Castro, "The large $N$ limit of Exceptional Jordan Matrix Models and M, F Theory" Journal of Geometry and Physics 57 (2007) 1941.

[13] M. Rios, "Jordan Algebras and Extremal Black Holes" hep-th/0703238. "The Geometry of Jordan Matrix Models" hep-th/0503015.

[14] L. Smolin, " The exceptional Jordan Algebra and the Matrix String" hepth/0104050

[15] M. Gunaydin, "Octonionc Hllbert Spaces, the Poincare Group and $S U(3)$ " J. Math. Phys 17, no. 10 (176) 1875. M. Gunaydin, C. Piron and H. Ruegg, "Moufang Plane and Octonionic Quantum Mechanics" Com. Math. Phys (1978).

[16] S. Catto, "Exceptional Projective Geometries and Internal Symmetries" hep-th/0302079.

[17] F.D. Smith Jr, " $E_{6}$, Strings, Branes and the Standard Model" [ CERN CDS EXT-2004-031 ]. Int. J. Theor. Phys 24, 155 (1985); Int. J. Theor. Phys 25 , 355 ( 1985 ) . "From Sets to Quarks" [ arXiv : hep-ph/9708379] . "The $D_{4}-D_{5}-E_{6}-E_{7}-E_{8}$ Model" [ CERN CDS EXT-2003-087 ] .

[18] K. Borchsenius, Phys. Rev D 13 (1976) 2707.

[19] J. Moffat and D. Boal, Phys. Rev D 11 (1975) 1375.

[20] S. Marques and C. Oliveira, J. Math. Phys 26 (1985) 3131. Phys. Rev D 36 (1987) 1716.

[21] C. Castro and M. Pavsic, Progress in Physics 1 (2005) 31. Phys. Letts B 559 (2003) 74. Int. J. Theor. Phys 42 (2003) 1693. 
[22] M. Pavsic," Spin gauge theory of Gravity in Clifford space : a realization of Kaluza Klein theory in 4-dim spacetime" gr-qc/0507053. " Int.J.Mod.Phys. A 21 (2006) 5905-5956. "Kaluza-Klein theory without extra dimensions : Curved Clifford space" Phys.Lett. B 614 (2005) 85-95, hep-th/0412255. "On a Unified theory of generalized Branes coupled to Gauge Fields, including the Gravitational and Kalb-Ramond Fields " hep-th/065126, to appear in Foundations Physics.

[23] G. Trayling, " A geometric approach to the Standard Model " hepth/9912231.

[24] I. R. Porteous Clifford algebras and Classical Groups Cambridge Univ. Press, 1995.

[25] M. Cederwall and J. Palmkvist, " The octic $E_{8}$ invariant" hep-th/0702024.

[26] S. Adler, "Further thoughts on Supersymmetric $E_{8}$ as family and grand unification theory" hep-ph/0401212. N. Baaklini, Phys. Lett 91 B (1980) 376. I. Bars and M. Gunaydin, Phys. Rev. Lett 45 (1980) 859. S. Konshtein and E. Fradkin, Pis'ma Zh. Eksp. Teor. Fiz 42 (1980) 575. M. Koca, Phys. Lett 107 B (1981) 73. R. Slansky, Phys. Reports 79 (1981) 1

[27] M. Gunaydin, K. Koepsell and H. Nicolai, "The Minimal Unitary Representation of $E_{8(8)}$ " Adv.Theor.Math.Phys. 5 (2002) 923-946, [arXiv : hepth/0109005]. M. Gunaydin, "Unitary Realizations of U-duality Groups as Conformal and Quasi Conformal Groups and Extremal Black Holes of Supergravity Theories" hep-th/0502235. M. Gunaydin and O. Pavlyk," Generalized spacetimes defined by cubic forms and the minimal unitary realizations of their quasi-conformal groups" JHEP 0508 (2005) 101, [arXiv : hep-th/0506010]. M. Gunaydin, K. Koepsell, and H. Nicolai, Comm. Math. Phys 221 (2001) 57. Adv. Teor. Math. Phys 5 (2002) 923.

[28] F. Toppan, "Quaternionic and octonionic spinors" hep-th/0503210. "Hermitian versus Holomorphic complex and quaternionic generalized supersymmetries of M theory, a classification" Phys.Rev. D 73 (2006) 084019, [arXiv : hep-th/0406022]. Z. Kuznetsova and F. Toppan, "Superalgebras of (split)division algebras and the split octonionic M-theory in (6,5)-signature" hepth/0610122.

[29] P. Ramond, Exceptional Groups and Physics, Plenary Talk delivered at Groupe 24 Conference, Paris, July 2002, [arXiv : hep-th/0301050].

[30] C. Hull, "Generalized Geometry for $M$ theory" hep-th/0701203.

[31] A. Kapustin and E. Witten," Electric-Magnetic Duality and the Geometric Langlands Program" hep-th/060415.

[32] M. Duff and S. Ferrara, " $E_{7}$ and the tripartite entanglement of seven qubits" quant-ph/0609227. 
[33] P. Levay, " Strings, black holes, the tripartite entanglement of seven quibits and the Fano plane" Phys. Rev. D 75 (2007) 024024, [arXiv : hepth/0610314].

[34] M. Gunaydin, A. Nietzke, B. Pioline and A. Waldron," BPS black holes, Quantum Attractor Flows and Automorphic Forms" Phys.Rev. D 73 (2006) 084019, [arXiv :hep-th/0512296].

[35] R. Foot and G. Joshi, Phys. Rev D 36 (1987) 1169.

[36] J. Baez, " The Octonions" Bull. Amer. Math. Soc. 39 (2002), 145-205, [arXiv : math.RA/0105155].

[37] S. Dangwal, P. Bisht and O. Negi, " Octonionic Gauge Formulation for Dyonic Fields" hep-th/0608061.

[38] C. Lassig and G. Joshi, " An Octonionic Gauge Theory" Chaos Solitons Fractals 7 (1996) 769, [arXiv : hep-th/9503189]. A. Ritz and G. Joshi," A Nonassociative Deformation of Yang-Mills Gauge Theory" Chaos Solitons Fractals 8 (1997) 835, [arXiv : hep-th/9508147].

[39] T. Ootsuka, E. Tanaka and E. Loginov, "Nonassociative Gauge Theory" [arXiv : hep-th/0512349] . E. Loginov," Analytic Loops and Gauge Fields" Nucl. Phys B 606 (2001) 636. "Multi-instantons in Higher Dimensions and Superstring Solitons" Symmetry, Integrability and Geometry : Methods and Applications 1 (2005)2, hep-th/0511262.

[40] J. A. Nieto, "Towards a Background Independent Quantum Gravity in Eight Dimensions" hep-th/0704.2769. "Towards an Ashtekar Formalism in Twelve Dimensions" hep-th/0506253.

[41] M. Gogberashvili, "Octonionic Geometry" Adv. in Appl. Clif. Alg., 15 (2005) 55, [arXiv :hep-th/0409173]. "Octonionic version of Dirac equation" Int.J.Mod.Phys. A21 (2006) 3513, [arXiv : hep-th/0505101].

[42] R. Held, I. Stavrov and B. Vankoten, " (Semi) Riemannian Geometry of (Para) Octonionic Projective Planes" [ arXiv : math.DG/0702631].

[43] L. Manivel, " Configurations of lines and models of Lie Algebras" math.RT/0507118.

[44] R. Friedman and J. Morgan, " Exceptional Groups and del Pezzo Surfaces" math.AG/0009155.

[45] S. De Leo, " Hypercomplex Group Theory" physics/9703033. S. De Leo and K. Abdel-Khalek, " Octonionic Representations of $G L(8, R)$ and $S L(4, C)$ " J. Math. Phys. 38 (1997) 582, [arXiv :hep-th/9607140[. "Octonionic Quantum Mechanics and Complex Geometry" Prog.Theor.Phys. 96 (1996) 823, [arXiv : hep-th/9609032]. 
[46] C. Manogue and T. Dray, " Octonionic Mobius Transformations" Mod. Phys. Lett. A 14 (1999) 1243, [arXiv : mat-ph/9905024].

[47] C. Castro, "A Chern-Simons $E_{8}$ Gauge Theory of Gravity, Grand Unification and Generalized Gravity in Clifford Spaces" submitted to the Int. Journal of Geom. Methods in Mod. Phys, March, 2007

[48] C. Castro, "On Chern-Simons (Super) Gravity, E 8 Yang-Mills and Polyvector valued Gauge Theories in Clifford Spaces" J. Math. Phys, 47, 112301 (2006)

[49] C. Castro, "On Generalized Yang-Mills Theories and Extensions of the Standard Model in Clifford (Tensorial) Spaces". Annals of Physics vol. 321 , no.4 (2006) 813-839. "Generalized p-forms Electrodynamics in Clifford Spaces Mod. Phys. Letts A 19 , no.1 (2004) 19-27.

[50] C. Castro, "On Modified Weyl-Heisenberg Algebras, Noncommutativity, Matrix-valued Planck Constant and QM in Clifford Spaces "Journal of Physics A : Math. Gen 39 (2006) 14205-14229.

[51] C. Castro, "Polyvector Super Poincare Algebras, $M, F$ theory algebras and Generalized Supersymmetry in Clifford Spaces" Int. Journal of Mod. Phys. A 21, no.10 (2005) 2149.

[52] C. Castro, "The Extended Relativity Theory in Born-Clifford Phase Spaces with a Lower and Upper Length Scale and Clifford Group Geometric Unification", Foundations of Physics 35, no.6 (2005) 971. Foundations of Physics 35 (2005) 971.

[53] C. Castro, "On Area Coordinates and QM in Yang's Noncommutative Spacetime with a lower and upper scale" Progress in Physics vol. 2 April (2006) 86-92

[54] J. Wess " Einstein-Riemann Gravity on Deformed Spaces" SIGMA 2 (2006), 089, [arXiv : hep-th/0611025]. P. Aschieri, M. Dimitrijevic, F. Meyer, and J. Wess, "Noncommutative Geometry and Gravity". Class.Quant.Grav. 23 (2006) 1883.

[55] G. Amelino-Camelia, Int. J. Mod. Phys D 11 (2002) 35. Int. J. Mod. Phys D 11 (2002) 1643. Phys. Let B 510 (2001) 255. J. Lukierski, A. Nowicki, H. Ruegg and V. Tolstoy, Phys. Letts B 264 (1991) 331. J. Lukierski, H. Ruegg and W. Zakrzewski, Annals of Phys. 243 (1995) 90.

[56] L. Boya, Mod.Phys.Lett. A21 (2006) 287-304.

[57] S. de Buyl, M. Henneaux and L. Paulot," Extended $E_{8}$ invariance of 11-dim Supergravity" JHEP 0602 (2006) 056, [arXiv : hep-th/0512292]. P. West, Class. Quan. Grav 18 (2001) 4443. V. A. Belinsky, I. M Khalatnikov and E. M. Lifshitz, Adv. Phys. 19 (1970) 525. 
[58] E. Cremmer, B. Julia and J. Scherk, Phys. Letts B 76 (1978 ) 409.

[59] T. Damour, A. Kleinschmidt and H. Nicolai, "Hidden Symmetries and the Fermionic Sector of 11-dim Supergravity " Phys.Lett. B 634 (2006) 319, [ arXiv : hep-th/0512163]. K. Koepsell, H.Nicolai and H. Samtleben, "An Exceptional Geometry for $D=11$ Supergravity ? " Class.Quant.Grav. 17 (2000) 3689, [ arXiv : hep-th/0006034] . H. Nicolai and H. Samtleben, "Maximal Gauged Maximal Supergravities in Three Dimensions" Phys.Rev.Lett. 86 (2001) 1686.

[60] J. Zanelli, "Lecture Notes on Chern-Simons (Super) Gravities", hepth/0502193. R. Troncoso and J. Zanelli," Gauge Supergravities for all Odd Dimensions" Int.J.Theor.Phys. 38 (1999) 1181, [arXiv : hep-th/9807029]. M. Hassaine, R. Troncoso and J. Zanelli, " Poincare invariant Gravity with local Supersymmetry as a gauge theory for the M algebra", Phys. Lett. B 596 (2004) 132, [ arXiv : hep-th/0306258]. F. Izaurieta, E. Rodriguez and P. Salgado," Euler Chern Simons Gravity from Lovelock Born Infeld Gravity " Phys. Lett. B 586 (2004) 397, [ arXiv : hep-th/0402208].

[61] D. Alekseevsky, V. Cortes, C. Devchand and A. Van Proeyen " Polyvector Super-Poincare Algebras " Commun. Math. Phys. 253 (2004) 385, [ arXiv : hep-th/0311107]. I. Rudychev, E. Sezgin, " Superparticles, p-form Coordinates and the BPS Condition" hep-th/9711128. I. Bars, C. Kounnas, "A new supersymmetry" hep-th/9612119. I. Bandos, J. Lukierski, "Generalized Superconformal Symmetries and Supertwistor Dynamics" hep-th/9912264.

[62] I. Frenkel and A. Todorov, "Complex counterpart of Chern-Simons-Witten theory and Holomorphic linking" math.AG/0502169. A. Borowiec, M. Ferraris and M. Francaviglia, J. Phys. A : Math. Gen 36 (2003) 2589. 